\title{
Norwalk Virus
}

National Cancer Institute

\section{Source}

National Cancer Institute. Norwalk Virus. NCI Thesaurus. Code C112363.

A species of non-enveloped and spherical viruses with a capsid with $T=3$ icosahedral symmetry in the Caliciviridae family and Norovirus genus. The genome is composed of single-stranded, positive-sense RNA. Norwalk viruses are found in humans and causes acute viral gastroenteritis. Norwalk viruses are transmitted via oral ingestion or fecal-oral contamination. 